Check for updates

Cite this: RSC Adv., 2018, 8, 37229

\title{
Joint effect of a combination of components from the fruit of Crataegus pinnatifida Bge. Var. major N.E. Br. and the root of Salvia miltiorrhiza Bge. with swimming on atherosclerosis in rats $\uparrow$
}

\author{
Leilei Gong, (DD a Yangyang Hao, ${ }^{\mathrm{b}}$ Xiaojie Yin, ${ }^{a}$ Lan Wang, ${ }^{a}$ Xiaojing Ma, ${ }^{a}$ Jun Cao, ${ }^{a}$ \\ Rixin Liang, ${ }^{* a}$ Fulong Liao ${ }^{\star a}$ and Jianyong Zhang ${ }^{c}$
}

Background: It has been recognized that exercise training can attenuate the progression of atherosclerosis (AS). The combined application of components from the fruit of Crataegus pinnatifida Bge. Var. major N.E. $\mathrm{Br}$. (CP) and the root of Salvia miltiorrhiza Bge. (SM) has been effective in the prevention and treatment of atherosclerosis. The present work aims to investigate the joint effects of extracts from the fruit of $\mathrm{CP}$ and the root of SM with swimming on atherosclerosis in rats. Method: To establish a rat atherosclerosis model, a combined method of partial ligation of the left common carotid artery leading to low shear stress and a high-fat diet was employed. Blood samples were collected to detect the blood lipid profile, which included total cholesterol ( $\mathrm{T}-\mathrm{CHO}$ ), low-density lipoprotein cholesterol (LDL-C), triglycerides (TG) and high-density lipoprotein cholesterol ( $\mathrm{HDL}-\mathrm{C})$; endothelial cytokines such as 6-keto-prostaglandin $\mathrm{F} 1 \alpha$ (PGF1 $\alpha$ ), endothelin (ET), thromboxane $B_{2}\left(T X B_{2}\right)$, plasminogen activator inhibitor-1 (PAI-1), and von

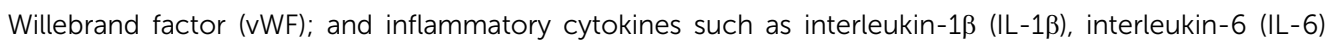
and interleukin-10 (IL-10). Finally, the common carotid arteries of the rats were removed to observe pathological changes via oil red $O$ staining, and the gene expression of t-PA, PAI-1, and vWF was assayed via real-time (RT) quantitative polymerase chain reaction (qPCR). Results: The joint effects of CPSM extract and swimming indicated significant interactions, including (1) decreased serum T-CHO, TG, and LDL-C; (2) decreased IL-6 and increased IL-10; (3) decreased TXB 2 , PAI-1 and VWF; three-dimensional analysis showed that gene expression of PAI-1 was inhibited, VWF gene expression was downregulated, and COX-1 gene expression was increased; and (4) decreased lipoprotein retention in the carotid artery wall. Conclusion: This research demonstrates that the combined therapy of CP and SM extracts with swimming can improve blood lipid levels and endothelial functions and attenuate the early signs of atherosclerosis in a rat model of atherosclerosis. The regulation of the gene expression of PAI, VWF and COX-1 may be the underlying cause of the effect. Methodologically speaking, three-dimensional surface plots of the joint effects of CPSM extract and swimming on parameters with quadratic fitting yielded a more accurate equation for describing the dose-response relationship in biomechanopharmacology. Such plots are likely worth using in pharmacology to quantify the effects induced by two medicinal factors.

Received 28th June 2018 Accepted 23rd October 2018

DOI: $10.1039 / \mathrm{c} 8 \mathrm{ra0} 5548 \mathrm{c}$ rsc.li/rsc-advances pathophysiology of AS, a pivotal factor, haemodynamic shear stress, should not be ignored. ${ }^{2}$ Emerging evidence has shown that atherosclerotic lesions only occur at the outer walls of the arterial branches and curvatures, where the local alteration of blood flow environments influences not only early plaque formation but also late development of AS., ${ }^{3,4}$ In vitro studies have revealed that the impact of blood flow on endothelial cells is mainly determined by two flow conditions: steady laminar flow and disturbed flow. The former is atheroprotective, while the latter creates an atheroprone location through thrombosis, inflammatory response and endothelial dysfunction, ${ }^{5}$ supporting the idea that disturbed flow is one of the primary characters in the procession of atherosclerosis.

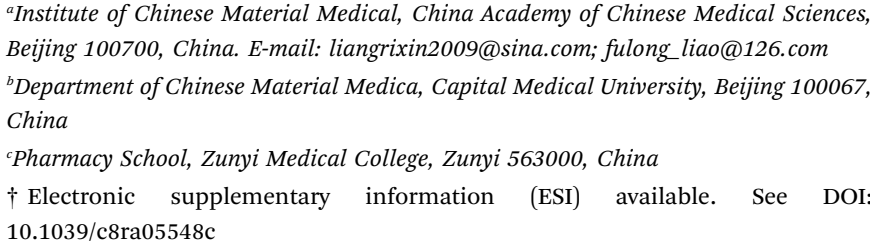

Atherosclerosis is a complex progressive vascular disease. Many endothelial dysfunction are strongly associated with the development of atherosclerosis (AS). ${ }^{1}$ However, in the 
The implication of lipoprotein retention into early atherosclerosis has been popularly accepted. Numerous studies proved that hypercholesterolemia, as an early risk factor, acts to promote the development of atherosclerosis via a mechanism of endothelial response to shear stress. A body of evidence demonstrated that LDL deposits at the surface of blood vessels are relevant to wall shear stress, and LDL accumulation within the vascular wall preferentially occurs in areas exposed to disturbed flow. ${ }^{6,7}$

In many ways, atherosclerosis is a chronic inflammatory disease. Russell Ross and John Glomset first described smooth muscle cell proliferation and migration in the formation of atherosclerotic lesions. Further studies found that atherosclerosis lesion formation is stimulated by inflammatory factors such as IL1, IL-6, IL-10 and TNF-1 $\alpha^{8}{ }^{8}$ More investigations have agreed that vascular inflammation and focal distribution of atherosclerotic lesions are associated with disturbed flow. A study from Caroline Cheng indicated that the expression of proatherogenic inflammatory mediators such as cytokine IL-6 and C-reactive protein is more prominent in the lower-shearstress regions. ${ }^{9}$

Endothelial cells are constantly subjected to mechanical forces in the form of shear stress generated by blood flow, and haemodynamic forces modulate endothelium functions by the expression of some genes associated with endothelial secretion. ${ }^{10}$ Previous in vitro studies indicated that fluid shear stress stimulates the expression of tissue plasminogen activator and reduces the secretion of plasminogen activator inhibitor type$1 .^{11,12}$ These changes in gene expression naturally lead to an impairment of homeostasis of endothelial functions. vWF is a large multimeric plasma glycoprotein and, as a biological marker for arterial and thrombotic diseases, is thought to be involved in atherosclerosis. ${ }^{13}$ The enhanced vasoconstriction of the aortic rings in atherosclerotic mice is mediated by COX-1 (not COX-2) via thromboxane A2 and ET-1, and COX-1 is closely associated with shear stress and $\mathrm{PGI}_{2} / \mathrm{TXA}_{2}$. In our previous study, we found that using ex vivo arterial perfusion system, the rabbit artery perfusion model was established under different flow conditions of shear stress (1.5-20 dyn $\mathrm{cm}^{-2}$ ), and the expression of COX-1 was increased and showed a positive correlation with shear stress. ${ }^{\mathbf{1 4}}$

Pharmacological therapy is a popular tragedy for the prevention and treatment of atherosclerosis. However, less is known about whether atherosclerosis can be intervened by no pharmacological methods. It has been gradually realized that physical exercise plays an important role in inhibiting atherosclerosis. Regular exercise alleviates damaged endothelial cells and suppresses atherosclerotic lesion formation. ${ }^{15,16}$ Shimada et al. found that swimming could reduce the severity of atherosclerosis in the carotid artery in apoE-deficient mice. Our previous study showed that the combination of a traditional Chinese medicine and swimming may prevent atherosclerosis through a synergistic interaction that improves blood circulation, haemorheological parameters, blood lipid levels and the vascular endothelium in a rat atherosclerosis model. ${ }^{\mathbf{1 6 , 1 7}}$

For many years, traditional Chinese medicine (TCM), by activating blood circulation, has been widely applied to prevent and treat cardiovascular diseases in China. CPSM extracts are an herbal pair. There are various reports on the biological activities and blood concentrations of its components, such as proanthocyanidin $\mathrm{B}_{2}$, salvianolic acid $\mathrm{B}$, and tanshinone $\mathrm{II}_{\mathrm{A}} \cdot{ }^{\mathbf{1 8 - 2 0}}$

In a previous study, we showed that aqueous extract of $\mathrm{CP}$ fruit attenuated atherosclerosis in rats by alleviating blood lipid levels, inflammation and endothelial dysfunction. ${ }^{21}$ In screening anti-atherosclerotic components, we found that the combination of effective components (proanthocyanidin $\mathrm{B}_{2}$, salvianolic acid $\mathrm{B}$, and tanshinone $\mathrm{II}_{\mathrm{A}}$ ) from the fruit of $\mathrm{CP}$ and the root of SM has anti-atherosclerotic activity, and the optimal ratio of each component was $84 \mathrm{mg} \mathrm{kg}^{-1}: 128 \mathrm{mg} \mathrm{kg}^{-1}: 25 \mathrm{mg}$ $\mathrm{kg}^{-1} \cdot{ }^{21}$

This study was designed to investigate the possible synergistic effect of exercise training with CPSM extract on atherosclerosis in rats and to explore the underlying mechanisms.

\section{Material and methods}

\subsection{Animal preparation}

The study was approved by the Research Ethics Committee of the Institute of Chinese Materia Medica, China Academy of Chinese Medical Science, Beijing, China. All animal studies were carried out in accordance with the guidelines and regulations for the care and use of laboratory animal of Center for Laboratory Animal Care, China Academy of Chinese Medical Science.

Fifty-one male Wistar rats (weight 200-220 g with a grade of specific pathogen-free) were purchased from Beijing Vital River Laboratory Animal Technology Co., Ltd. (SCXK2012-0001; Beijing, China). They were allocated into sixteen treatment groups ( $n=3$ each) and one sham-operated group $(n=3)$. Rats were housed at $25^{\circ} \mathrm{C}$ and $40 \%$ relative humidity and a $12 \mathrm{~h}$ light/dark cycle. They received humane care and had free access to food and water. All animal experiments were permitted by the Laboratory Animal Ethics Committee of the Institute of Chinese Material Medical, China Academy of Chinese Medical Sciences (Beijing, China). All animals were treated according to international ethics requirements.

\subsection{Surgical procedure}

To establish an animal model of early atherosclerosis, the common carotid artery (CCA) of the rats was ligated. The ligation of the CCA referred to the methods of Shi Fang Ding, ${ }^{22}$ Douglas $\mathrm{Nam}^{23}$ and Fang Hua. ${ }^{24}$ First, the rats were anaesthetized using $10 \%$ chloral hydrate at $0.3 \mathrm{~g} \mathrm{~kg}^{-1}$ dosage via intraperitoneal injection. Second, the rats were kept in a supine position. At the midline of the neck, a surgical incision was made, and the CCA and external carotid artery bifurcation were explored and carefully isolated to avoid damaging the vessels or recurrent laryngeal nerve. Next, a segment of suture (5-0) was placed around the CCA, and a sterile acupuncture needle with an outer diameter of $0.30 \mathrm{~mm}$ (Wuxi Jiajian Medical Instrument Company, Wuxi, China, lot: 20150310) was kept under the suture parallel to the CCA. The needle and the CCA were bundled together using the suture at a distance of $1.5 \mathrm{~cm}$ from 
the bifurcation. Then, the needle was removed, while the suture was kept around the CCA. As a result, the formation of a CCA ligation was finished. After the carotid surgery, gentamicin sulphate $\left(3.5 \mathrm{ml} \mathrm{kg}^{-1}\right)$ was administered in a single intramuscular injection. The sham-operated group was treated in the same way with no ligation of the artery. After the surgical operation, the rats were housed in a cage at $28{ }^{\circ} \mathrm{C}$ for $3 \mathrm{~h}$, then transferred to the animal care room and administered a normal diet. Afterwards, $6 \times 10^{5} \mathrm{IU} \mathrm{kg}^{-1}$ vitamin $\mathrm{D}_{3}$ (Shanghai General Pharmaceutical Co., Ltd., China, lot: 130203) was intraperitoneally injected once per day for 3 days in all groups except for the sham-operated group.

\subsection{Administration}

One week after ligation of the CCA, the rats in the shamoperated group were routinely given a normal diet, the rats in the treatment groups were fed a high-fat diet $1 \%$ cholesterol, $0.2 \%$ pig bile salts, $10 \%$ lard, $10 \%$ egg yolk powder, $78.8 \%$ basal diet, purchased from Beijing Keao Xieli Feed Co., LTD, China). The treatment lasted for 8 weeks in all groups, except the shamoperated group.

\subsection{Swimming design}

This study employed a factorial design for two factors, swimming and dose of CPSM extract. The four levels of each factor were as follows: swimming (no swimming, $10 \mathrm{~min}$ per day, $20 \mathrm{~min}$ per day or $40 \mathrm{~min}$ per day) and CPSM extract (0, $118.5 \mathrm{mg}, 237 \mathrm{mg}$ or $474 \mathrm{mg} \mathrm{kg}^{-1}$ body weight in rat per day). Sixteen groups of combined treatment were included in the $4 \times$ 4 factorial design. For describing the quantitative effect of joint CPSM extract and swimming on some measured parameters, a three-dimensional surface plot with quadratic fitting was drawn.

CPSM extracts were given to the rats orally at $0,118.5 \mathrm{mg}$, $237 \mathrm{mg}$ or $474 \mathrm{mg} \mathrm{kg}^{-1}$ body weight once per day, six days per week. The amount of swimming was determined according to You $^{\mathbf{1 6}}$ with a modulation. The rats were placed in a plastic swimming pool $(100 \mathrm{~cm} \times 60 \mathrm{~cm} \times 80 \mathrm{~cm})$ containing water at $35 \pm 1{ }^{\circ} \mathrm{C}$. The exercise duration was gradually increased by 10 min per day until the maximum swimming duration of 20 or $40 \mathrm{~min}$ was reached, which the rats were then subjected to 6 days per week. The swimming phases were supervised to ensure that every rat was exercising during the implementation stage of the swimming protocol. The swim training was done till the end of the eighth week.

\subsection{CPSM extract formulation}

CPSM extract were composed of aqueous extract of $\mathrm{CP}$ fruit (CPA), aqueous extract of SM (SMA) and liposoluble extract of SM (SML). All the compounds were extracted from SM and CP fruit by the Chemistry Department of Institute of Chinese Materia Medica, according to the method of Jianyong Zhang. ${ }^{21}$ By high-performance liquid chromatography (HPLC) analysis, it was proved that the main component of SM aqueous extract was salvianolic acid $\mathrm{B}$; the main component of SM liposoluble extract was tanshinone $\mathrm{II}_{\mathrm{A}}$; the main component of CPA was proanthocyanidin $\mathrm{B}_{2}$. CPSM extract was prepared as a mixture at the ratio of $128: 84: 25$ of CPA : SMA : SML.

\subsection{Biochemical parameters}

After 8 weeks of intervention, all rats were fasted through the night of the last day of the 8th week and anaesthetized using $10 \%$ chloral hydrate at a dose of $0.3 \mathrm{~g} \mathrm{~kg}^{-1}$ via intraperitoneal injection on the first day of the 9th week. Blood samples were collected through the abdominal aorta. A $1.5 \mathrm{ml}$ whole blood sample was collected using $7.5 \%$ disodium ethylene diamine tetraacetic acid (EDTA) and aprotinin (Solarbio, China, lot: 115B0330) as anti-coagulants and then centrifuged at $3000 \mathrm{rpm}$ for $15 \mathrm{~min}$ at $4{ }^{\circ} \mathrm{C}$ to determine the contents of IL-1 $\beta$, 6-ketoprostaglandin $\mathrm{F}_{1 \alpha}$ (6-keto-PGF $\left.\mathrm{P}_{1 \alpha}\right)$ and thromboxane $\mathrm{B}_{2}\left(\mathrm{TXB}_{2}\right)$, which is a stable metabolite of thromboxane $\mathrm{A}_{2}\left(\mathrm{TXA}_{2}\right)$. Because $\mathrm{TXA}_{2}$ is unstable and rapidly metabolized, $\mathrm{TXB}_{2}$ was used as a measurement of platelet activation in the plasma. The additional $3 \mathrm{ml}$ whole-blood sample without anticoagulation was centrifuged at $3000 \mathrm{rpm}$ for $15 \mathrm{~min}$ at $4{ }^{\circ} \mathrm{C}$, and the serum was retained for determination of other biochemical parameters.

\subsection{Determination of serum lipid profile}

Blood lipid profile was measured with a multi-function microplate reader (SpectraMax Plus 384, Molecular Devices Corporation, USA) using commercially available kits according to the manufacturer's instructions, including total cholesterol ( $\mathrm{T}$ $\mathrm{CHO}$ ), triglyceride (TG), low-density lipoprotein-cholesterol (LDL-C) and high-density lipoprotein-cholesterol (HDL-C). The four testing kits were all purchased from Nanjing Jiancheng Bioengineering Institute, China, with lot numbers of 20160607 , 20160608, 20160606 and 20160604.

\subsection{Determination of endothelial function factors}

The concentrations of plasma 6-keto-PGF $1 \alpha$ and $\mathrm{TX}-\mathrm{B}_{2}$ were assayed with a gamma radioimmunoassay (RIA) counter (DFM96, Hefei Zhongcheng Electromechanical Co., Ltd., China) using commercially available kits. The three kits were purchased from Beijing Northern Bio-technology Research Institute, China, with the same lot number of 20160620. The concentrations of serum plasminogen activator inhibitor-1 (PAI-1) and von Willebrand factor (vWF) were measured by ELISA using commercially available kits according to the manufacturer's instructions. The three kits were purchased from Shanghai Fan Ke Industrial Co., Ltd., China, with the same lot number of 20160620 .

\subsection{Analysis of inflammatory cytokines}

The concentration of plasma IL- $1 \beta$ was determined by RIA using a commercially available kit (Beijing Northern Bio-technology Research Institute, China, lot: 20160620) according to the manufacturer's instructions. Serum IL-6 and IL-10 concentrations were measured by ELISA using commercially available kits according to the manufacturer's protocols. The two kits were purchased from Shanghai Fan Ke Industrial Co., Ltd., China, with the same lot number of 20160620. 
Table 1 Selected genes, primers and amplicon characteristics

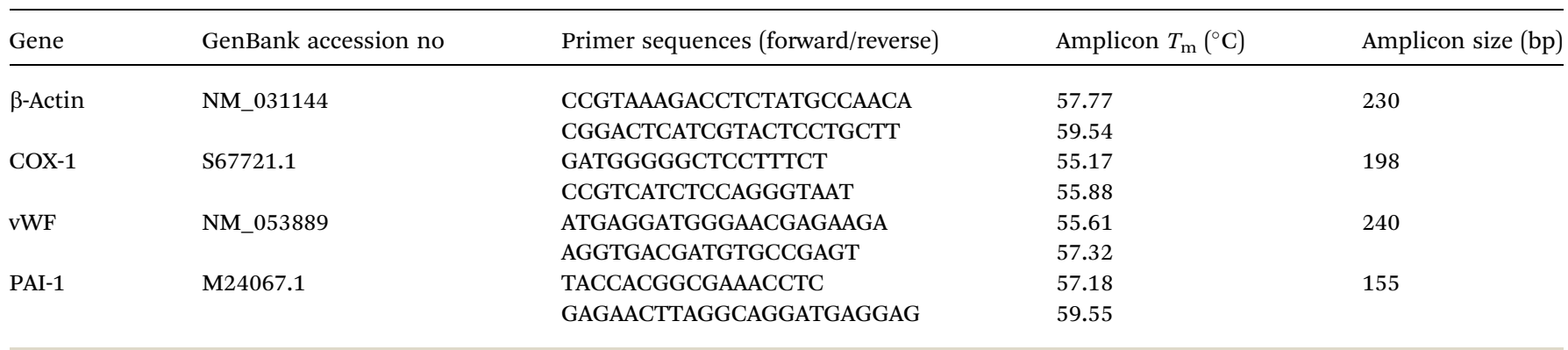

\subsection{Histopathological assessment of the carotid artery}

After blood was withdrawn, the common carotid arteries $1.5 \mathrm{~cm}$ from the ligation on either side were harvested immediately and fixed with Tissue OCT-Freeze Medium (Sakura Finetek Co., Ltd., Japan, lot: 4583) and then flashfrozen in liquid nitrogen. Carotid artery frozen sections (4 $\mu \mathrm{m}$ ) produced by a freezing microtome (Leica CM 1900, Leica Microsystems, Germany) were stained with oil red $\mathrm{O}$ and then observed under an optical microscope (Leica DM 2500 LED, Leica Microsystems, Germany). The images were obtained at $\times 200$ magnification, and histopathological evaluations were performed using 3-5 sections from different locations of the carotid artery. The oil red $\mathrm{O}$ staining area was calculated using ImageJ 1.52a (National Institutes of Health, USA).

\subsection{RNA isolation}

After blood was withdrawn, the right common carotid arteries (RCCAs) $1.5 \mathrm{~cm}$ from the ligation were harvested immediately. Each blood vessel was flash-frozen in liquid nitrogen with an RNase-free EP tube and stored at $-80{ }^{\circ} \mathrm{C}$ or directly processed for RNA extraction.

\subsection{Quality control and cDNA synthesis}

Microbiology and aseptic techniques were followed to create and maintain an RNase-free environment and to avoid RNase contamination, during or after extraction. Samples were finely ground for efficient cell lysis in liquid nitrogen using a ball mill (Mixer Mill MM 400, Retsch Technology, Germany). Total RNA of the aorta samples was extracted using the Eastep Super kit (Promega Corporation, USA, lot: 0000187133) according to the manufacturer's instructions. Total RNA samples were purified using an RNA clean kit (Beijing TIANGEN Biotech Co., Ltd., China, lot: 03806) according to the manufacturer's instructions. The RNA concentration and purity were determined using a Biospec-nano spectrophotometer (Shimadzu Corporation, Japan). Only RNA samples with a 260/280 ratio between 1.9 and 2.1 and a 260/230 ratio higher than 2.0 were used for subsequent analyses. Then, total RNA was reverse-transcribed using the FastQuant RT Kit (Beijing TIANGEN Biotech Co., Ltd., China, lot: P4415) according to the manufacturer's instructions.

\subsection{Determination of gene expression by RT-qPCR}

RT-qPCR was performed for PAI-1, COX-1 and vWF with the reference gene $\beta$-actin. Primer pairs were designed in silico based on selected sequences by using Primer Premier 5.0 software (Premier Biosoft, USA) (see Table 1). The specificity of all primer sequences was first tested by homology searches in the nucleotide database (NCBI, nucleotide BLAST). All primers were custom-ordered from a commercial supplier (Beijing RuiBiotech Co., Ltd., China). RT-qPCR was performed in 96-well plates with an ABI 7300 Real-Time PCR System and 7300 System Software (Applied Biosystems, USA) by using a KAPA SYBR FAST qPCR Kit (Kapa Biosystems, USA, lot: KK4601). The amplification reaction mixture (total volume of $20 \mu \mathrm{l}$ ) contained $10 \mu \mathrm{l}$ of $2 \times$ SYBR Fast qPCR Master Mix, $0.4 \mu \mathrm{l}$ of each primer $(10 \mu \mathrm{m}), 1$ $\mu \mathrm{l}$ of template cDNA, $0.4 \mu \mathrm{l}$ of $50 \times$ Rox High and dd- $\mathrm{H}_{2} \mathrm{O}$ to the final volume. Each reaction mix was subjected to the following conditions: $95{ }^{\circ} \mathrm{C}$ for $3 \mathrm{~min} ; 40$ cycles of $95{ }^{\circ} \mathrm{C}$ for $3 \mathrm{~s}, 60{ }^{\circ} \mathrm{C}$ for $30 \mathrm{~s}$. The data of relative gene expression obtained from the 7300 System Software were analysed via the $2^{-\Delta \Delta C_{t}}$ method. ${ }^{25}$

\subsection{Statistical analysis}

Statistical analysis was performed with SPSS version 21.0 (SPSS, Chicago, IL, USA). All data are expressed as the mean \pm SD. Comparisons of factor levels between the sham group and model group were analysed by using the independent-sample $t$ test. The GLM univariate procedure in SPSS 21.0 was performed for two-way ANOVA to test for synergism, and a Bonferroni post hoc test was used to show the differences between each pair of factor levels. A value of $P<0.05$ was considered statistically significant. Three-dimensional surface plot of joint effects of CPSM extract and swimming with quadratic fitting on parameters was completed by Statistica 6.0 software.

\section{Results}

\subsection{Determination of blood lipoprotein}

Compared with the sham-operated group, serum T-CHO (ranging from $0.65 \pm 0.10$ to $2.39 \pm 0.19 \mathrm{mmol} \mathrm{L}^{-1}$ ), LDL-C (from $0.65 \pm 0.12$ to $1.11 \pm 0.08 \mathrm{mmol} \mathrm{L}^{-1}$ ) and TG (0.69 \pm 0.15 to $1.08 \pm 0.13 \mathrm{mmol} \mathrm{L}^{-1}$ ) values in the model group were significantly higher $(P<0.05)$, while the level of HDL-C (from $1.17 \pm 0.01$ to $\left.0.79 \pm 0.05 \mathrm{mmol} \mathrm{L}^{-1}\right)$ was significantly lower $(P<$ 0.05) (see Fig. 1A). 

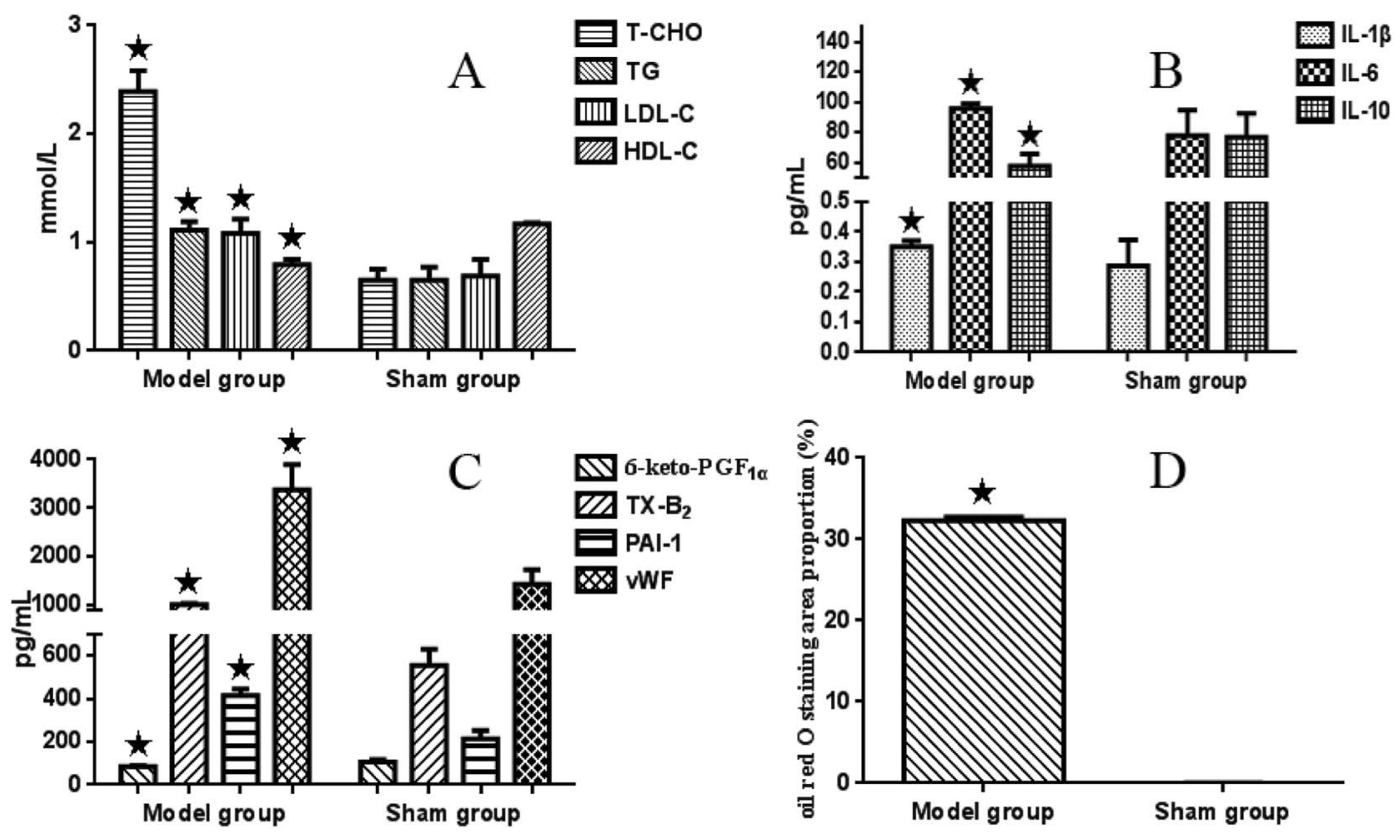

Fig. 1 Bar graphs of serum lipoproteins, inflammatory cytokines, and endothelial factors in the model group and sham-operated group. All data are expressed as the mean \pm SD. Comparisons between the sham group and model group were analysed using the independent-sample $t$-test. A value of $P<0.05$ was considered statistically significant.

There were significant interaction effects between CPSM extract and swimming on lowering serum T-CHO $(F=2.801$ and $P<0.015)$, TG $(F=3.982$ and $P<0.002)$ and LDL-C $(F=2.412$ and $P<0.032)$ levels. The combination of $474 \mathrm{mg} \mathrm{kg}^{-1} \mathrm{CPSM}$ extract with 20 min swimming exerted a marked action (T-CHO, from $2.39 \pm 0.19$ to $1.54 \pm 0.23 \mathrm{mmol} \mathrm{L}^{-1}$; TG, from $1.08 \pm 0.13$ to $0.42 \pm 0.13 \mathrm{mmol} \mathrm{L}^{-1}$; LDL-C, from $1.11 \pm 0.08 \mathrm{mmol} \mathrm{L}^{-1}$ to $0.42 \pm 0.10 \mathrm{mmol} \mathrm{L}^{-1}$ ), which indicated that the combination of

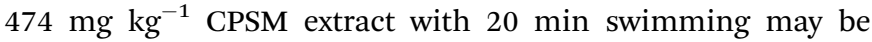
optimum and helpful in improving blood lipoprotein. However, no significant interaction effect between CPSM extract and swimming on HDL-C was found ( $F=0.381, P=0.936$ ). Only swimming for different times showed a significant effect on HDL-C $(F=8.647, P<0.001)$, suggesting that swimming alone is more effective than CPSM extract on regulating HDL-C (see Table 2).

\subsection{Measurements of inflammatory factors}

Plasma IL-1 $\beta$ and serum IL- 6 values in the model group were significantly higher (ranging from $0.287 \pm 0.086$ to $0.351 \pm 0.02$ pg ml ${ }^{-1}$ and from $78.10 \pm 16.41$ to $95.68 \pm 3.24 \mathrm{pg} \mathrm{ml}^{-1}$, respectively) $(P<0.05)$ than those in the sham-operated rats, and serum IL-10 (from $76.94 \pm 15.76$ to $57.61 \pm 8.16 \mathrm{pg} \mathrm{ml}^{-1}$ ) was markedly lower $(P<0.05)$ (see Fig. 1B).

There were apparent interaction effects between CPSM extract and swimming on serum IL-6 $(F=8.757, P<0.001)$. The $237 \mathrm{mg} \mathrm{kg}^{-1}$ CPSM extract combined with $10 \mathrm{~min}$ swimming showed a decreased action (ranging from $95.68 \pm 3.24$ to 63.53 $\left.\pm 13.90 \mathrm{pg} \mathrm{ml}^{-1}\right)$. An interaction effect on IL-10 was also seen $(F$ $=4.267, P=0.001)$. The group composed of $474 \mathrm{mg} \mathrm{kg}^{-1} \mathrm{CPSM}$ extract and $10 \mathrm{~min}$ swimming had significantly increased IL-10 $\left(57.61 \pm 8.16\right.$ to $\left.129.53 \pm 6.22 \mathrm{pg} \mathrm{ml}^{-1}\right)$. While there was no interaction on plasma IL-1 $\beta(F=1.620, P=0.152)$, both CPSM extract with different dosages $(F=0.933, P=0.436)$ and swimming for different times $(F=2.348, P=0.091)$ had no influence on IL-1 $\beta$. The above results may suggest that the interaction of $237 \mathrm{mg} \mathrm{kg}^{-1}$ and $474 \mathrm{mg} \mathrm{kg}^{-1}$ CPSM extract and 10 min swimming on decreasing IL-6, a proinflammatory cytokine, and increasing IL-10, an anti-inflammatory cytokine, should be beneficial for the production of inflammatory factors in atherosclerosis (see Table 3).

The effect of CPSM extract and swimming on inflammatory factors could similarly be described by the three-dimensional surface plot with quadratic fitting (see Fig. 2):

$$
\begin{aligned}
\text { Serum IL-6 }\left(\mathrm{pg} \mathrm{ml}^{-1}\right) & =86.892+29.0244 x+0.1044 y-7.2145 x^{2} \\
& -0.666 x y+0.0189 y^{2}(\text { see Fig. } 2 \mathrm{~A})
\end{aligned}
$$

Plasma IL-10 $\left(\mathrm{pg} \mathrm{ml}^{-1}\right)=65.7727-16.0395 x+2.1724 y$

$$
+3.0306 x^{2}-0.6141 x y-0.0351 y^{2} \text { (see Fig. 2B) }
$$

Serum IL-1 $\beta\left(\mathrm{pg} \mathrm{ml}^{-1}\right)=0.303-0.025 x-0.0025 y+0.0044 x^{2}$ $+0.0003 x y+4.7866 E-5 y^{2}$ (see Fig. 2C)

\subsection{Detection of endothelial factors}

In the model rats, plasma 6-keto-PGF ${ }_{1 \alpha}$ was lower (ranging from $106.02 \pm 12.08$ to $86.10 \pm 3.60 \mathrm{pg} \mathrm{ml}^{-1}$ ) and $\mathrm{TX}-\mathrm{B}_{2}$ was significantly higher (from $554.30 \pm 75.17$ to $1013.05 \pm 24.45 \mathrm{pg} \mathrm{m}^{-1}$ ) than those in the sham-operated rats $(P<0.05)$. Meanwhile, serum PAI-1 (from $214.58 \pm 38.25$ to $415.39 \pm 31.07 \mathrm{pg} \mathrm{ml}^{-1}$ ) and vWF (from $1433.82 \pm 297.22$ to $3370.98 \pm 520.31 \mathrm{pg} \mathrm{ml}^{-1}$ ) were obviously increased $(P<0.05)$ in the model group compared with the sham-operated group (see Fig. 1C). As seen 
Table 2 Blood lipoproteins in different treatment groups (mean \pm SD) $(n=3)^{a}$

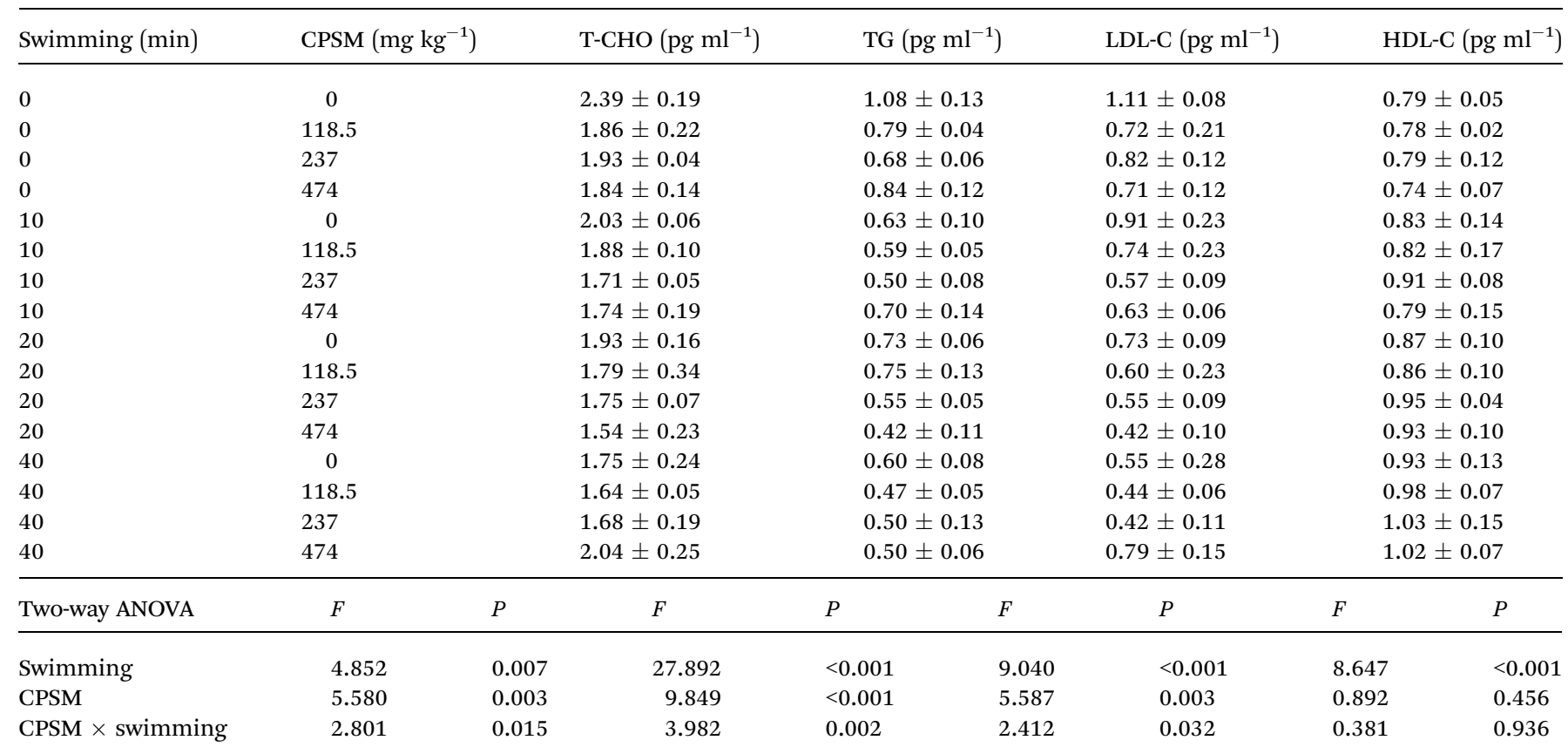

${ }^{a}$ All data are expressed as the mean \pm SD. The GLM procedure was applied for a two-way ANOVA to test for synergy. Comparisons between each pair of factor levels were analysed using a Bonferroni post hoc test. A value of $P<0.05$ was considered statistically significant.

in Table 4, there still existed significant interactive effects between CPSM extract and swimming on decreasing $\mathrm{TXB}_{2}(F=$ 3.804, $P=0.002)$. The combined treatment of $474 \mathrm{mg} \mathrm{kg}^{-1}$ CPSM extract with 20 min swimming exhibited a marked effect on $\mathrm{TXB}_{2}$ (from $956.90 \pm 158.26$ to $308.77 .78 \pm 94.87 \mathrm{pg} \mathrm{ml}^{-1}$ ).
But and interaction between CPSM extract and swimming on increasing plasma 6-keto-PGF ${ }_{1 \alpha}$ did not occur $(F=0.405, P=$ 0.923). Only $20 \mathrm{~min}$ swimming raised 6-keto-PGF ${ }_{1 \alpha}$ from 86.10 \pm 3.60 to $167.71 \pm 18.51 \mathrm{pg} \mathrm{ml}^{-1}(F=14.473, P<0.001)$. There were also significant interactive effects between CPSM extract

Table 3 Inflammatory cytokines and oil red O staining area proportion in different treatment groups $(\operatorname{mean} \pm \mathrm{SD})(n=3)^{a}$

\begin{tabular}{|c|c|c|c|c|c|c|c|}
\hline Swimming (min) & CPSM $\left(\mathrm{mg} \mathrm{kg}^{-1}\right)$ & & $\mathrm{IL}-1 \beta\left(\mathrm{pg} \mathrm{ml}^{-1}\right)$ & IL-6 $\left(\mathrm{pg} \mathrm{ml} \mathrm{m}^{-1}\right)$ & $\mathrm{IL}-10\left(\mathrm{pg} \mathrm{ml}^{-1}\right)$ & \multicolumn{2}{|c|}{ Area proportion (\%) } \\
\hline 0 & 0 & & $0.351 \pm 0.020$ & $95.68 \pm 3.24$ & $57.61 \pm 8.16$ & \multicolumn{2}{|c|}{$32.29 \pm 0.41$} \\
\hline 0 & 118.5 & & $0.278 \pm 0.014$ & $119.78 \pm 30.39$ & $121.50 \pm 33.34$ & \multicolumn{2}{|c|}{$1.24 \pm 0.29$} \\
\hline 0 & 474 & & $0.265 \pm 0.038$ & $88.28 \pm 13.74$ & $130.39 \pm 12.91$ & \multicolumn{2}{|c|}{$3.13 \pm 0.43$} \\
\hline 10 & 0 & & $0.267 \pm 0.060$ & $81.77 \pm 8.17$ & $52.50 \pm 22.97$ & \multicolumn{2}{|c|}{$10.20 \pm 1.14$} \\
\hline 10 & 118.5 & & $0.244 \pm 0.030$ & $83.80 \pm 3.46$ & $77.13 \pm 10.63$ & \multicolumn{2}{|c|}{$0.37 \pm 0.15$} \\
\hline 20 & 0 & & $0.244 \pm 0.058$ & $72.70 \pm 24.33$ & $118.94 \pm 17.98$ & \multicolumn{2}{|c|}{$11.49 \pm 2.35$} \\
\hline 20 & 118.5 & & $0.285 \pm 0.037$ & $139.18 \pm 3.09$ & $126.39 \pm 9.35$ & \multicolumn{2}{|c|}{$1.69 \pm 0.71$} \\
\hline 20 & 237 & & $0.249 \pm 0.023$ & $99.65 \pm 9.01$ & $120.06 \pm 19.20$ & \multicolumn{2}{|c|}{$0.04 \pm 0.01$} \\
\hline 20 & 474 & & $0.274 \pm 0.050$ & $65.24 \pm 26.35$ & $129.53 \pm 6.22$ & \multicolumn{2}{|c|}{$0.03 \pm 0.01$} \\
\hline 40 & 0 & & $0.252 \pm 0.026$ & $131.18 \pm 11.01$ & $69.39 \pm 14.06$ & \multicolumn{2}{|c|}{$0.15 \pm 0.01$} \\
\hline 40 & 118.5 & & $0.262 \pm 0.026$ & $101.94 \pm 3.45$ & $117.22 \pm 10.37$ & \multicolumn{2}{|c|}{$0.45 \pm 0.10$} \\
\hline Swimming & 2.348 & 0.091 & 5.189 & 0.005 & $<0.001$ & 238.003 & 0.000 \\
\hline CPSM & 0.933 & 0.436 & 5.507 & 27.173 & $<0.001$ & 450.841 & 0.000 \\
\hline $\mathrm{CPSM} \times$ swimming & 1.620 & 0.152 & 8.757 & $<0.001$ & $<0.001$ & 92.550 & 0.000 \\
\hline
\end{tabular}

${ }^{a}$ All data are expressed as the mean \pm SD. The GLM procedure was applied for a two-way ANOVA to test for synergy. Comparisons between each pair of factor levels were analysed using a Bonferroni post hoc test. A value of $P<0.05$ was considered statistically significant. 

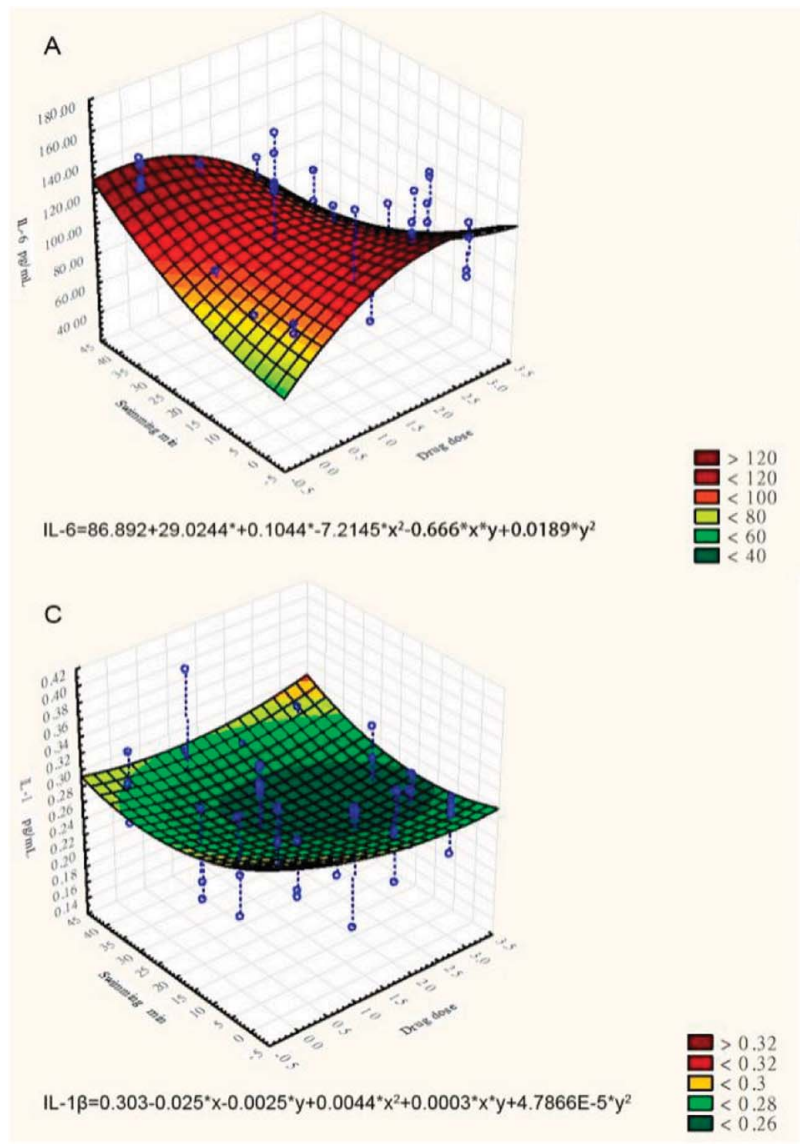

B
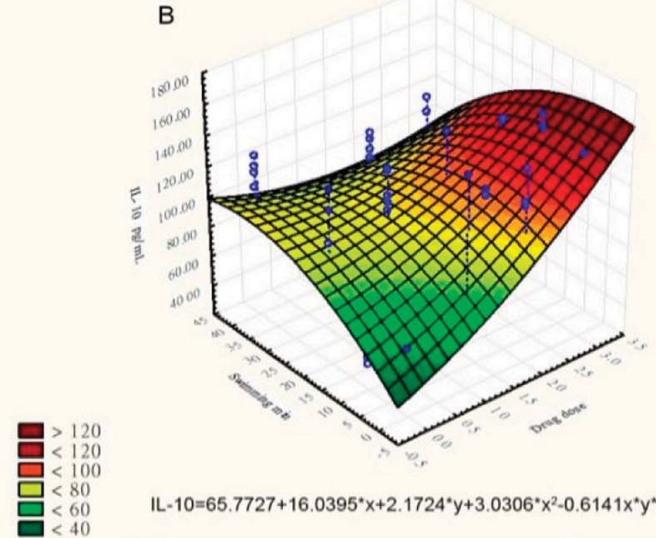

IL- $10=65.7727+16.0395^{*} x+2.1724^{*} y+3.0306^{*} x^{2}-0.6141 x^{*} y^{*}-0.0351^{*} y^{2}$

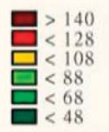

Fig. 2 Three-dimensional surface plots of the effects of TCPSM extract and swimming on inflammatory factors. (A) Serum IL-6 (pg $\mathrm{ml}^{-1}$ ); (B) plasma IL-10 (pg ml$\left.{ }^{-1}\right)$; (C) serum IL-1 $\beta\left(\mathrm{pg} \mathrm{ml}^{-1}\right)$.

and swimming on changing serum PAI-1 $(F=13.348, P<0.001)$ and $\mathrm{vWF}(F=8.524, P<0.001)$. vWF was lowered from $2630.53 \pm$ 290.08 to $1191.90 \pm 64.79 \mathrm{pg} \mathrm{ml}^{-1}$ by the combination of $237 \mathrm{mg} \mathrm{kg}^{-1}$ CPSM extract and $10 \mathrm{~min}$ swimming, and PAI-1 was lowered from $415.39 \pm 31.07$ to $247.72 \pm 82.19 \mathrm{pg} \mathrm{m}^{-1}$ by the combination of $237 \mathrm{mg} \mathrm{kg}^{-1}$ CPSM extract and 0 min swimming. In brief, the results may demonstrate that the combined treatment of CPSM extract and swimming affected the secretion of $\mathrm{TXB}_{2}$, PAI-1 and vWF from endothelial cells, which may have contributed to the improvement of endothelial functions.

We used three-dimensional surface plots to describe the joint effects of CPSM extract and swimming on endothelial factors (see Fig. 3). The influences of CPSM extract and exercise on those factors could also be described by quadratic equations:

$$
\begin{aligned}
& \text { Serum PAI-1 }\left(\mathrm{pg} \mathrm{ml}^{-1}\right)=432.9663-0.4847 x+7.2939 y \\
&+0.0007 x^{2}-0.0047 x y-0.1144 y^{2}(\text { see Fig. 3A })
\end{aligned}
$$

Plasma TXB $2\left(\mathrm{pg} \mathrm{ml}^{-1}\right)=1118.5637-1.0236 x-6.3612 y$

$$
+0.0011 x^{2}+5.8870 \times 10^{3} x y-0.1935 y^{2}(\text { see Fig. 3B) }
$$

Serum vWF $\left(\mathrm{pg} \mathrm{ml}^{-1}\right)=3323.017-2.7669 x-57.5248 y$

$$
+0.0038 x^{2}-0.006 x y+0.9879 y^{2} \text { (see Fig. 3C) }
$$

\subsection{Histopathological assessment}

The sham-operated group showed no histopathological changes. Compared with the sham-operated group, oil red Ostained histological sections in the model group showed large amounts of lipid droplets under the common carotid artery intima, and even some lipid droplets merged to form lipid pools, thus accumulating as lipid plaques. Based on the histopathological assessment, the animal model was in the initial state of atherosclerosis. The combination of CPSM extract and swimming suppressed lipoprotein retention (see Fig. 4-6). The oil red O staining area proportion was $32.29 \pm 0.41 \%$ in the model group (Fig. 1D). An interaction between CPSM extract and swimming was found $(F=92.550, P<0.000)$. The treatment groups of $474 \mathrm{mg} \mathrm{kg}^{-1}$ and $20 \mathrm{~min}$ swimming and of $237 \mathrm{mg}$ $\mathrm{kg}^{-1}$ and 40 min swimming markedly reduced the staining area proportion from $32.29 \pm 0.41 \%$ to $0.03 \pm 0.01 \%$ and $0.03 \pm$ $0.01 \%$, respectively. These results may show that the joint intervention of CPSM extract and swimming could lessen the staining area proportion of lipoprotein retention, at least partly attenuating plaque formation in early atherosclerosis (see Table 3: area proportion).

\subsection{Expression of PAI-1, COX-1 and vWF genes}

The relative expression levels of three genes (PAI-1, COX-1, and $\mathrm{vWF}$ ) were quantified via the $2^{-\Delta \Delta C_{\mathrm{t}}}$ method and are presented 
Table 4 Endothelial function factors in different treatment groups (mean \pm SD) $(n=3)^{a}$

\begin{tabular}{|c|c|c|c|c|c|}
\hline Swimming (min) & CPSM (mg kg $\left.{ }^{-1}\right)$ & 6-keto-PGF ${ }_{1} \alpha\left(\mathrm{pg} \mathrm{ml}^{-1}\right)$ & $\mathrm{TX}^{-\mathrm{B}_{2}}\left(\mathrm{pg} \mathrm{ml} \mathrm{m}^{-1}\right)$ & PAI-1 $\left(\mathrm{pg} \mathrm{ml}^{-1}\right)$ & $\mathrm{vWF}\left(\mathrm{pg} \mathrm{ml} \mathrm{l}^{-1}\right)$ \\
\hline 0 & 0 & $86.10 \pm 3.60$ & $1013.05 \pm 24.45$ & $415.39 \pm 31.07$ & $3370.98 \pm 520.31$ \\
\hline 0 & 118.5 & $89.49 \pm 11.54$ & $1097.32 \pm 48.76$ & $408.65 \pm 3.53$ & $3139.61 \pm 218.08$ \\
\hline 0 & 474 & $111.39 \pm 11.62$ & $771.93 \pm 191.52$ & $540.74 \pm 35.28$ & $3119.72 \pm 383.80$ \\
\hline 10 & 0 & $105.22 \pm 10.51$ & $1008.67 \pm 291.55$ & $483.90 \pm 61.29$ & $2630.53 \pm 290.08$ \\
\hline 10 & 118.5 & $103.13 \pm 34.49$ & $909.13 \pm 223.35$ & $550.44 \pm 97.73$ & $2741.71 \pm 177.03$ \\
\hline 20 & 0 & $154.30 \pm 34.61$ & $956.90 \pm 158.26$ & $453.94 \pm 58.53$ & $2950.73 \pm 301.59$ \\
\hline 20 & 118.5 & $154.77 \pm 48.88$ & $983.67 \pm 349.84$ & $460.99 \pm 11.30$ & $2079.09 \pm 385.65$ \\
\hline 20 & 237 & $141.68 \pm 11.95$ & $555.89 \pm 216.95$ & $498.75 \pm 90.95$ & $1989.76 \pm 571.72$ \\
\hline 20 & 474 & $167.71 \pm 18.51$ & $308.77 \pm 94.87$ & $343.34 \pm 41.55$ & $2612.74 \pm 270.84$ \\
\hline 40 & 0 & $145.44 \pm 24.72$ & $582.07 \pm 93.35$ & $643.85 \pm 52.96$ & $2385.94 \pm 270.99$ \\
\hline 40 & 118.5 & $164.52 \pm 27.10$ & $423.43 \pm 111.73$ & $261.38 \pm 44.42$ & $2001.27 \pm 152.75$ \\
\hline Swimming & 17.473 & $<0.001$ & $<0.001$ & 0.072 & 24.183 \\
\hline CPSM & 1.861 & 0.156 & 6.033 & 0.002 & 0.001 \\
\hline $\mathrm{CPSM} \times$ swimming & 0.405 & 0.923 & 13.348 & $<0.001$ & $<0.001$ \\
\hline
\end{tabular}

${ }^{a}$ All data are expressed as the mean \pm SD. The GLM procedure was applied for a two-way ANOVA to test for synergy. Comparisons between each pair of factor levels were analysed using a Bonferroni post hoc test. A value of $P<0.05$ was considered statistically significant.

by the three-dimensional surface plot (see Fig. 7). The joint effects of CPSM extract and swimming on the relative expression levels of PAI-1, COX-1, and vWF mRNA could also be described by quadratic equations:

Relative expression level of PAI-1 mRNA $=1.8424-0.0027 x+$ $0.0626 y+3.3317 x^{2} \times 10^{-6}-4.5679 x y \times 10^{-6}-0.0012 y^{2}$

(see Fig. 7A)

Relative expression level of COX-1 mRNA $=0.7134+0.0024 x+$ $0.0663 y-3.4175 x^{2} \times 10^{-6}-2.213 x y \times 10^{-6}-0.0014 \times y^{2}$

(see Fig. 7B)

Relative expression level of vWF mRNA $=2.4335-0.0027 x-$ $0.0461 y+3.7854 x^{2} \times 10^{-6}-1.539 x y \times 10^{-5}+0.0008 y^{2}$

(see Fig. 7C)

where $x$ represents the dosage of CPSM extract and $y$ represents the intensity of swimming.

Based on the equations, 0-10 min exercise training combined with $237 \mathrm{mg} \mathrm{kg}^{-1}$ CPSM extract inhibited the expression of PAI-1, and 10-30 min exercise training combined with $237 \mathrm{mg} \mathrm{kg}^{-1}$ CPSM extract downregulated vWF while upregulating COX-1.

\section{Discussion}

In the present study, there existed interactive effects between CPSM extract and exercise training in atherosclerosis rats. The interactions included (1) decreased serum lipids such as TCHO, TG, and LDL-C and attenuated pathological signs of early atherosclerosis, as seen by lower oil red $\mathrm{O}$ staining area proportion and lipoprotein retention; (2) inflammatory alleviation, with decreased IL-6 and increased IL-10; (3) improvement of endothelial function, with reduction of $\mathrm{TXB}_{2}, \mathrm{PAI}-1$ and vWF; (4) according to three-dimensional analysis, inhibited PAI-1 gene expression, downregulated vWF gene expression, increased cyclooxygenase-1 (COX-1) gene expression.

\subsection{Role of shear stress in the procession of atherosclerosis in rats}

Shear stress induced by blood flow plays a critical role in the development of atherosclerosis. Atherosclerosis preferentially occurs in regions of disturbed flow. The most direct evidence linking disturbed flow to atherosclerosis is from suitable animal models. ${ }^{26,27}$ This work employed a rat model of early atherosclerosis induced by a combination of low shear stress status induced by partial ligation of the common carotid artery followed by feeding with a high-fat diet and injection of vitamin $\mathrm{D}_{3}$ and ovalbumin. The animal model group showed pathogenic characteristics of early atherosclerosis. By 12 weeks, hyperlipidaemia (T-CHO ranging from $2.39 \pm 0.19$ to $1.54 \pm$ $0.23 \mathrm{mmol} \mathrm{L}^{-1}$; TG, from $1.08 \pm 0.13$ to $0.42 \pm 0.13 \mathrm{mmol} \mathrm{L}^{-1}$; LDL-C, from $1.11 \pm 0.08 \mathrm{mmol} \mathrm{L}^{-1}$ to $0.42 \pm 0.10 \mathrm{mmol} \mathrm{L}^{-1}$ ) and lipid retention in the common carotid artery wall (oil red $\mathrm{O}$ staining area proportion $32.29 \pm 0.41 \%$ in the model group) were found. Meanwhile, IL-1 $\beta$, IL-6, TX-B ${ }_{2}$, PAI- 1 and vWF were increased and IL-10 and 6-keto-PGF1 $\alpha$ decreased in the model group. These results suggest that the animal model was successfully reproducible, shear stress induced early atherosclerosis in the presence of some risk factors, and the 


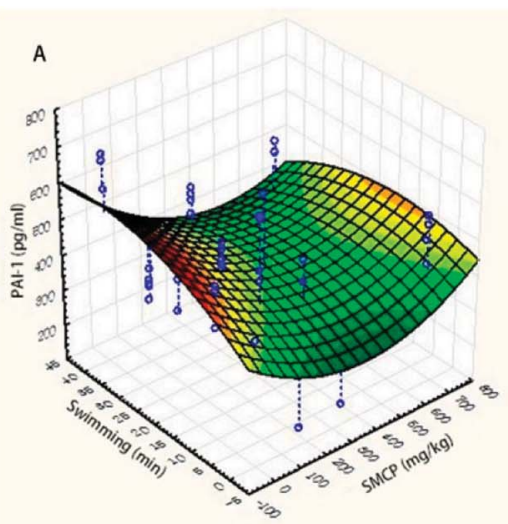

PAI $1=432.9663-0.4847^{*} x+7.2939^{*} y+0.0007^{*} x^{2}-0.0047^{*} x^{*} y-0.1144^{*} y^{2}$

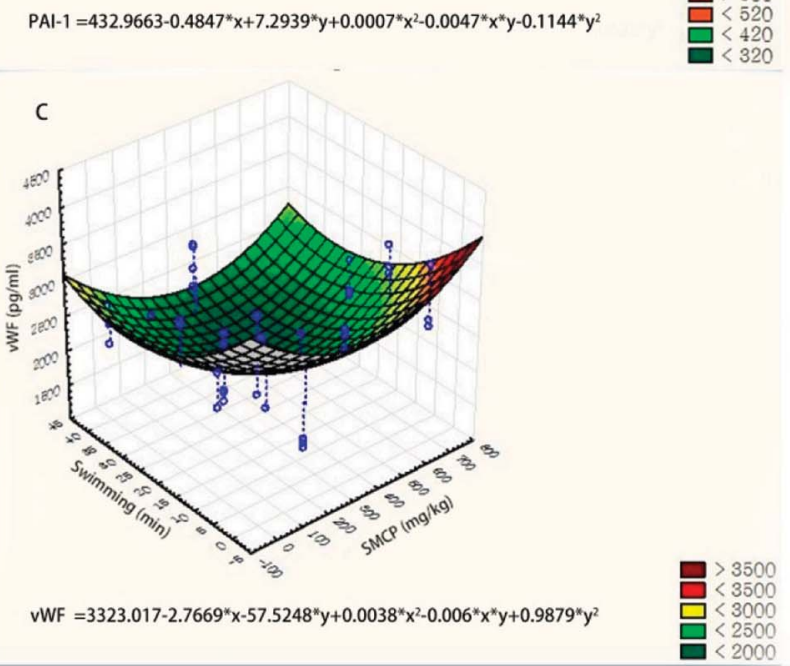

B

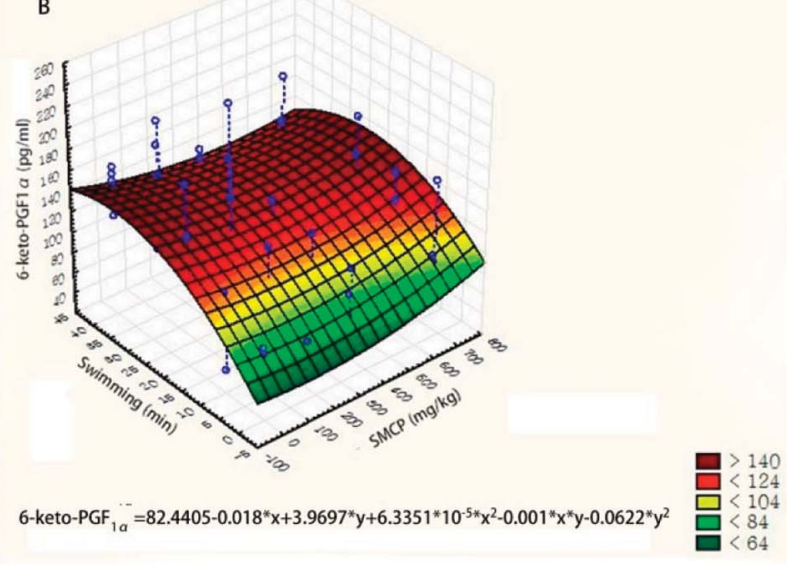

Fig. 3 Three-dimensional surface plots of effects of CPSM extract and swimming on endothelial factors. (A) Serum PAI-1 (pg ml ${ }^{-1}$ ); (B) plasma $\mathrm{TXB}_{2}\left(\mathrm{pg} \mathrm{m} \mathrm{m}^{-1}\right)$; (C) serum vWF $\left(\mathrm{pg} \mathrm{ml}^{-1}\right)$.

mechanical force of blood flow appears to be a key factor in the initiation of atherosclerosis in rats.

\subsection{The synergistic effects of combined CPSM extract with swimming in atherosclerosis rats}

Atherosclerosis is a complex disease caused by various risk factors, which make finding effective prevention and treatment methods challenging. Modern medical strategy greatly depends on drugs such as statins and interventional therapy. However, in Chinese medicine, the concept that diseases are prevented by promoting blood circulation has been followed since ancient times, so Chinese herbs that activate blood circulation and eliminate stasis are widely applied clinically to prevent and treat cardiovascular diseases. Alternatively, the potential effects of regular physical training on atherosclerosis have been widely appreciated. In this study, we applied a combined treatment of CPSM extract and swimming in a rat atherosclerosis model. For rats, swimming is a natural behaviour that can be exploited in a quantitative way under proper experimental controls and avoids the emotional arousal induced by physical exercise.

Atherosclerotic plaque often forms in curved arteries with low shear stress and oscillation. ${ }^{28}$ In the earliest stages of atherosclerosis, cholesterol accumulates, especially high levels of LDL-C, bringing about the formation of foam cells and accelerating the growth of atherosclerotic lesions ${ }^{29}$ indicating that local haemodynamic factors can promote lipoprotein retention. In the present study, the levels of LDL-C, TG and TC were measured. Significant interactive effects between CPSM extract and swimming on decreasing LDL-C $(F=2.412, P=$ $0.032)$, TG $(F=3.982, P=0.002)$ and TC $(F=2.801, P=0.015)$ were found. Meanwhile, the vascular wall lipid deposits were attenuated, which suggested that the combination of CPSM extract and swimming exerted a significant effect in decreasing blood lipid concentrations and pathological characteristics of early atherosclerosis. Hyperlipidaemia triggers the initiation of atherosclerosis. Our results indicate that the joint action of CPSM extract combined with swimming can reduce blood lipids, which may be a potential approach to delay the development of atherosclerosis.

Inflammatory responses evoked by low shear stress are considered a key process in the development of atherosclerotic plaque. Investigations indicate that the interleukin (IL) family is closely involved in the inflammatory precession of atherosclerosis, and members of the IL family predominately regulate endothelial activities by producing some chemoattractant proteins, such as monocyte chemotactic proteins and adhesion molecules. Among them, IL-10 is a prototypical antiinflammatory cytokine. IL-6 is an independent risk factor for 


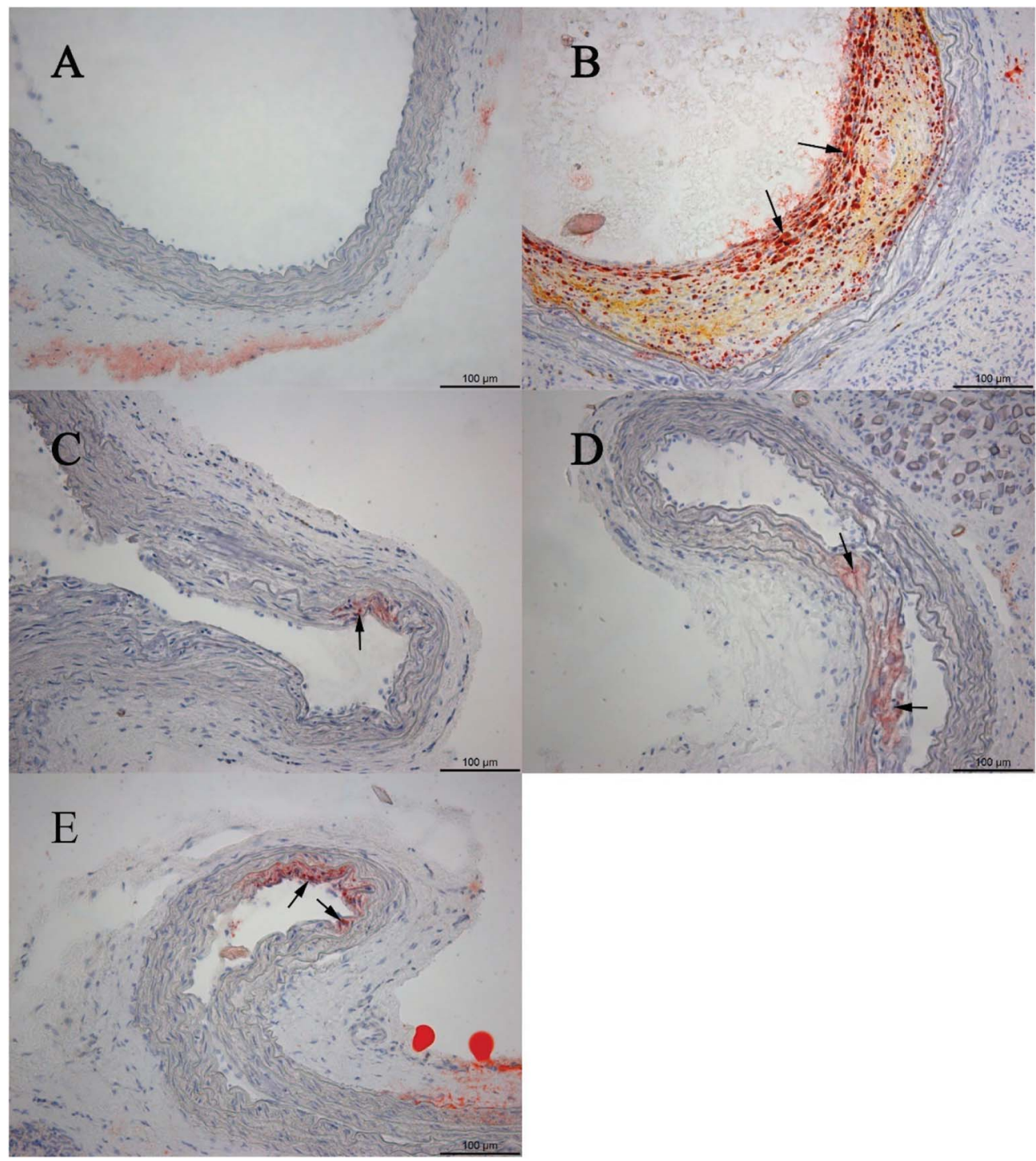

Fig. 4 Oil red O-stained histological sections. (A) Example of oil red O-stained sections of an aortic segment in the sham group; (B) example section of a model rat without any treatment; (C) example section of the rats treated with $118.5 \mathrm{mg} \mathrm{kg}^{-1}$ CPSM extract per day; (D) example section of a rat treated with $237 \mathrm{mg} \mathrm{kg}^{-1}$ CPSM extract per day; (E) example section of a rat treated with $474 \mathrm{mg} \mathrm{kg}^{-1}$ CPSM extract per day.

the promotion of atherosclerosis, as a direct proatherosclerotic mediator. ${ }^{30}$ Recent studies indicated that IL-1 expression is increased in human coronary arteries affected by atherosclerosis and is also associated with arterial inflammation and oxidative stress. ${ }^{31}$ Therefore, the correlation between haemodynamic shear stress and inflammation in atherosclerosis deserve more attention. The results from the present study show that the combined application of swimming and CPSM extract increased IL-10 and decreased IL-6, which was characterized by a significant interaction $(F=4.267, P<0.001 ; F=$ 8.757, $P<0.001)$. Similar results were found from swimming alone. These results show that the joint action of quantitative exercise training and CPSM extract could have an active influence on the inflammatory responses in a rat atherosclerosis model by modulating the induction of IL-1, IL-6 and IL-10. Further, the atherosclerosis development was delayed.
Many studies revealed that ECs are highly sensitive to shear stress and undergo a variety of alterations in function. The endothelium maintains vascular homeostasis through modulating secretion of endothelial factors, including thrombus formation genes. Endothelial dysfunction caused by alterations of shear stress can stimulate the production of some bioactive markers that contribute to atherosclerosis lesion formation. ${ }^{32}$

PAI-1 is the major inhibitor of t-PA. As a risk factor for ischaemic cardiovascular events, the elevated PAI-1 promotes the development of atherosclerotic plaque by promoting thrombus formation..$^{33}$ Platelet activation and aggregation are closely associated with atherosclerosis procession by the initial response of the vessel wall to mechanical injuries. $\mathrm{TXA}_{2}$, as a potent chemoattractant, accelerates platelet activation and has great importance in the formation of atherosclerotic plaque. TXA $\mathrm{T}_{2}$ is unstable and is rapidly metabolized $\mathrm{TXB}_{2}$, which is commonly considered a parameter determining platelet 


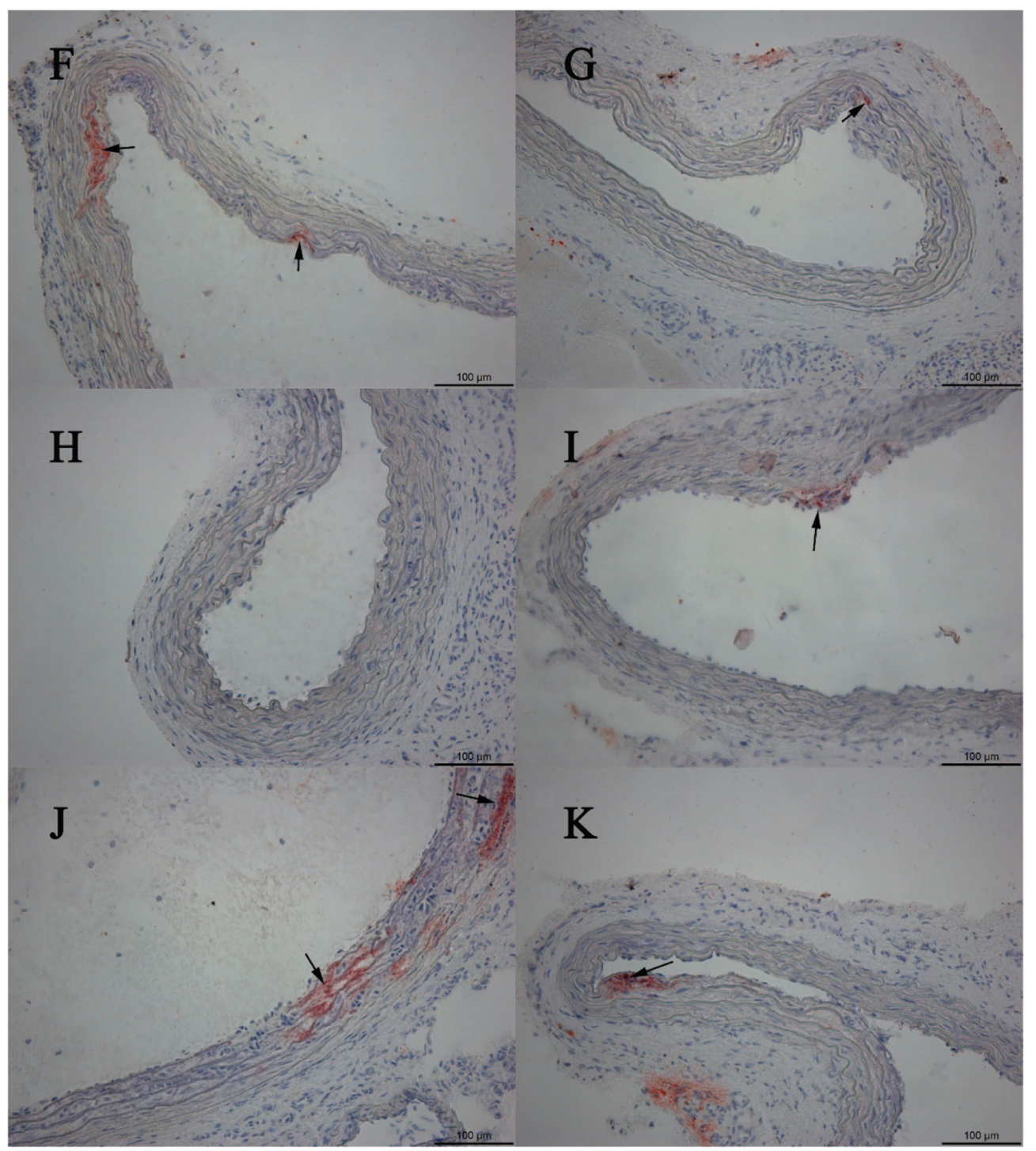

Fig. 5 Oil red O-stained histological sections. (F) Example section of a rat treated with 10 min swimming per day; (G) example section of a rat treated with $118.5 \mathrm{mg} \mathrm{kg}^{-1}$ CPSM extract and 10 min swimming per day; $(\mathrm{H})$ example section of a rat treated with $237 \mathrm{mg} \mathrm{kg}^{-1}$ CPSM extract and 10 min swimming per day; (I) example section of a rat treated with $474 \mathrm{mg} \mathrm{kg}^{-1}$ CPSM extract and 10 min swimming per day; (J) example section of a rat treated with 20 min swimming per day; $(K)$ example section of a rat treated with $118.5 \mathrm{mg} \mathrm{kg}^{-1}$ CPSM extract and 20 min swimming per day.

activation..$^{34} \mathrm{vWF}$ is involved in atherosclerosis, primarily by mediating the attachment of platelets to exposed tissue, resulting in the acceleration of atherosclerotic plaque formation. ${ }^{35}$ Our results show that the joint effect of swimming combined with CPSM extract decreased vWF $(F=13.348, P<$ $0.001), \mathrm{TXB}_{2}(F=3.804, P=0.002)$ and PA-1 $(F=8.524, P<$ $0.001)$. These findings may further explain why the endothelial dysfunction induced by abnormal shear stress can be improved by some interventions such as exercise training and CPSM extract. Inhibition of platelet aggregation might be helpful in abolishing the initial response of vessel walls to injury induced by shear stress.

Endothelial cell functions are altered by haemodynamic forces, which usually change the expression of genes related to endothelial functions. ${ }^{36}$ Over 40 genes have been identified as being regulated by shear stress in endothelial cells and play a key role in atherosclerosis. Evidence indicated that shear stress dependent-genes contribute to the proliferation and differentiation of SMCs, thrombosis and modulation of the inflammatory responses. The measurement of the expression of such genes is important for the understanding of pathological mechanisms and application of therapeutic strategies in atherosclerosis. ${ }^{37-39}$ The present study was designed to investigate the expression of genes related to thrombosis and dissolution, such as PAI-1, COX-1 and VWF, and observe the influences of exercise combined with CPSM extract on the above genes' expression. The results showed that the combination of swimming for 10-30 min and CPSM extract at a medium dosage depressed the expression of PAI- 1 and vWF but upregulated COX-1, according to three-dimensional surface plots. These 


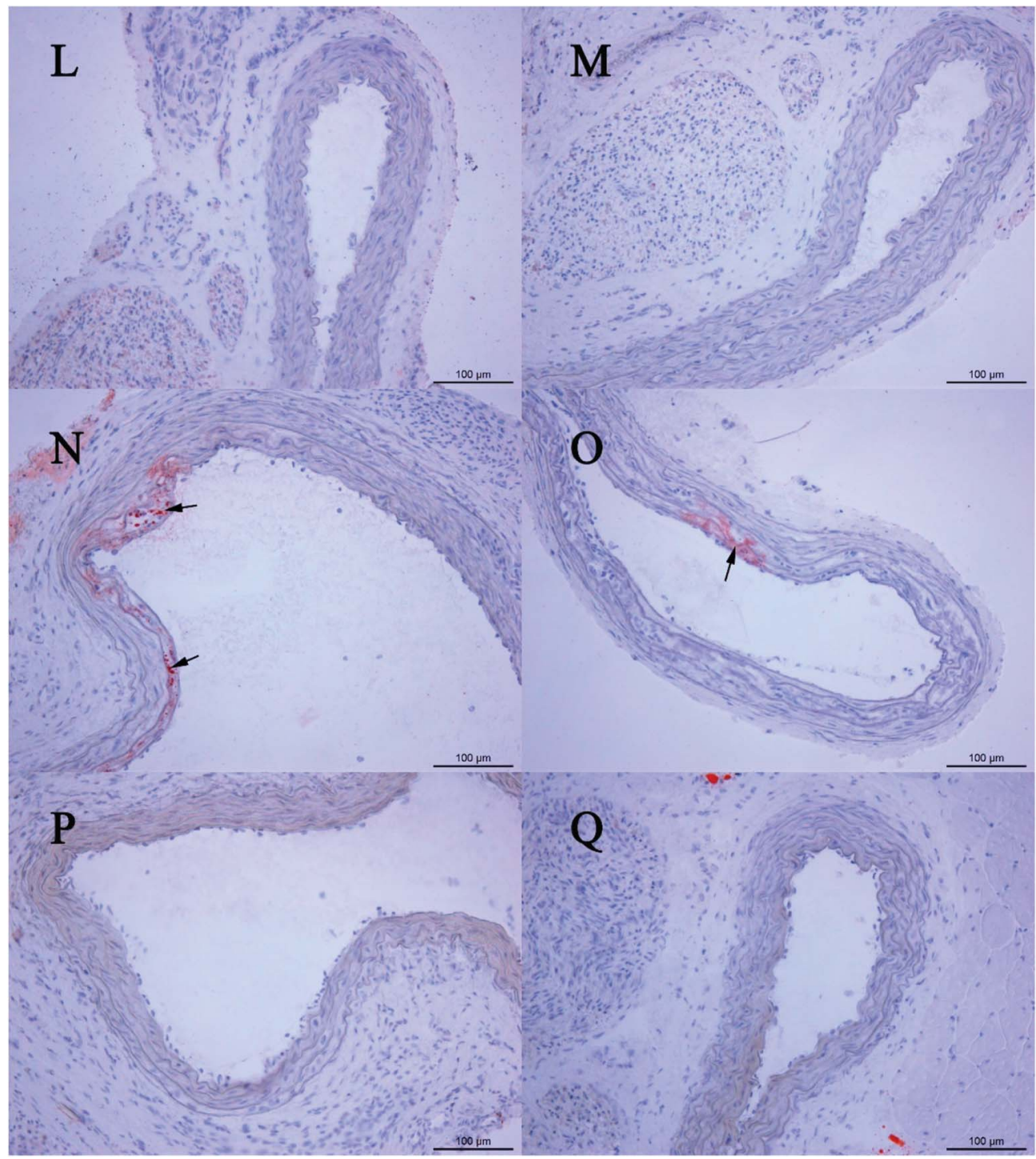

Fig. 6 Oil red O-stained histological sections. (L) Example section of a rat treated with $237 \mathrm{mg} \mathrm{kg}^{-1}$ CPSM extract and 20 min swimming per day; (M) example section of a rat treated with $474 \mathrm{mg} \mathrm{kg}^{-1}$ CPSM extract and 20 min swimming per day; (N) example section of a rat treated with 40 min swimming per day; (O) example section of a rat treated with $118.5 \mathrm{mg} \mathrm{kg}^{-1}$ CPSM extract and 40 min swimming per day; (P) example section of a rat treated with $237 \mathrm{mg} \mathrm{kg}^{-1}$ CPSM extract and 40 min swimming per day; (Q) example section of a rat treated with 474 mg kg ${ }^{-1}$ CPSM extract and 40 min swimming per day. Arrows point to the lipid droplets. The example section (B) of the model rat could be recognized as the initial state of atherosclerosis. The combination of CPSM extract and swimming (C-Q) suppressed the pathological changes to some degree. Magnification $\times 200$.

data suggest that the combination of exercise and CPSM extract could regulate the expression of genes associated with thrombosis and dissolution, by which endothelial functions such as the secretion of PAI- $1, \mathrm{vWF}$, and $\mathrm{TXB}_{2}$ were improved and atherosclerosis lesion severity was alleviated.

\subsection{Concluding remarks and study limitation}

Atherosclerosis is a multifactorial disease. The prevention and treatment afforded by modern medicine are undoubtedly effective, but they are also costly, and patient compliance is always put at risk by side effects. Therefore, alternative therapies, particularly natural products, are increasingly explored. CPSM extract is a combination of extracts from the fruit of CP and the root of SM and has been widely applied in the treatment of atherosclerosis. In China, the efficacy and safety of CPSM have been popularly accepted. Exercise training can protect against atherosclerosis. The mechanisms of this effect may be correlated with the suppression of inflammatory responses and improvement of endothelial dysfunction. The shear-regulated gene expression of endothelium is stimulated to maintain the homeostasis of vascular structure and function even under haemodynamic shear stress. As a new borderline discipline, biomechanopharmacology ${ }^{40}$ has focussed on the joint effects of biomechanical and pharmacological interventions in the prevention and treatment of cardiovascular diseases. An increasing amount of evidence indicates that exercise training may act on inflammation, endothelial dysfunction, and lipoprotein retention. Strikingly, regular exercise may become 
A

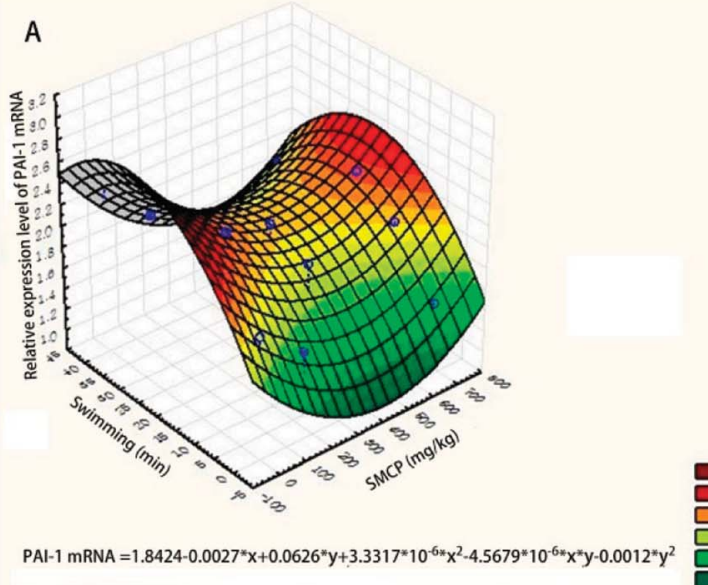

B

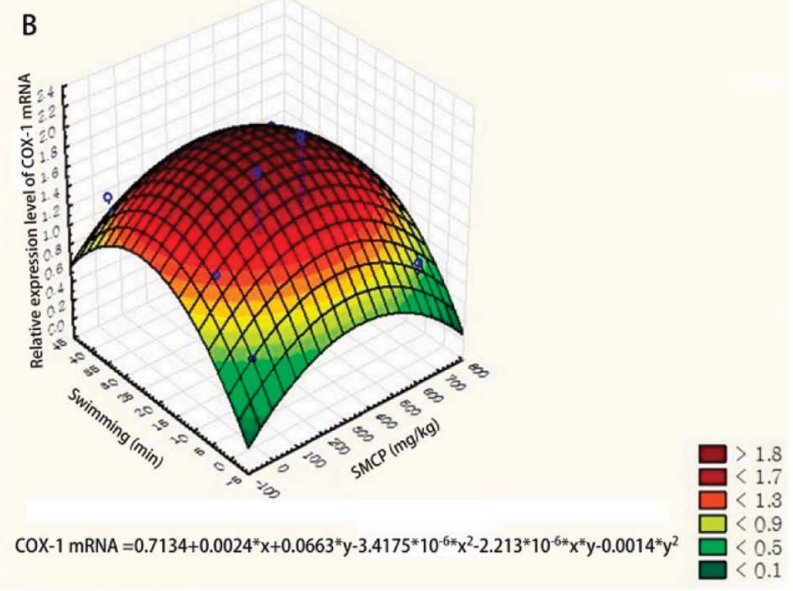

C

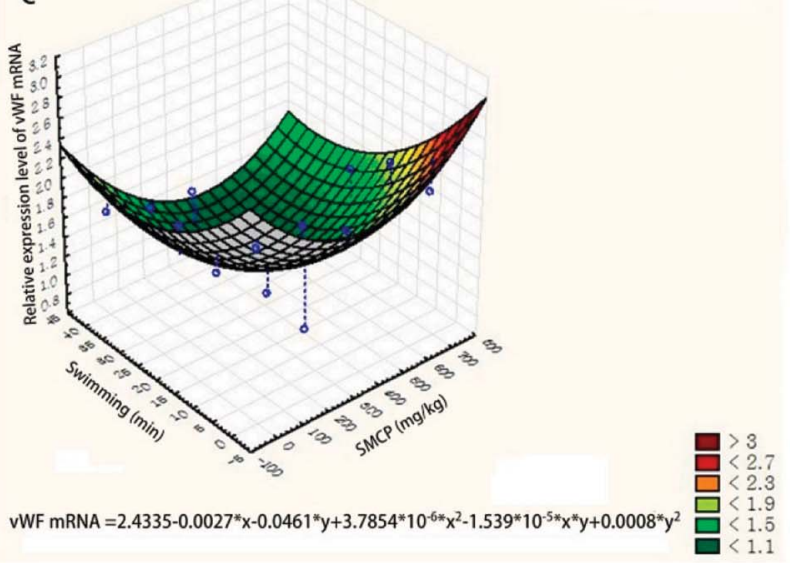

Fig. 7 Three-dimensional surface plot of effects of CPSM extract and swimming on relative expression levels of genes. (A) Relative expression level of PAI-1 mRNA; (B) relative expression level of COX-1 mRNA; (C) relative expression level of vWF mRNA.

a potential strategy for the prevention and treatment of atherosclerosis. Our results reveal that CPSM extract with middle and high dosages combined with swimming for $10 \mathrm{~min}$ to 40 min showed significant interactive effects on improving early atherosclerosis and endothelial dysfunction, as well as decreasing blood lipids and inflammatory markers. Meanwhile, the ameliorated gene expression of PAI-1, COX-1 and vWF may be involved in the mechanisms of the synergistic reaction. These results are special importance because they point out that the prevention and treatment of atherosclerosis can be achieved by synergistic effects derived from exercise and CPSM extract, rather than an intervention of synthetic drugs. In conclusion, a biomechanopharmacologically tailored exercise should be emphasized because it could provide a new insight into the prevention and treatment of atherosclerosis. For a deeper understanding of the definite actions and mechanisms induced by the combination of exercise and medicines, more experimental studies will be required. The results gained from clinical observations will be convincing.

In summary, this study indicates that the combination of CPSM (a medicinal factor) and swimming (a physical exercise) may prevent atherosclerosis through synergistic effects on blood lipids, inflammatory factors, vascular endothelial function and plaque formation in a rat atherosclerosis model. The mechanism may be associated with the regulation of the PAI, vWF and COX-1 genes.

\section{Author contributions}

All the authors have approved the manuscript and agree with submission to your esteemed journal.

\section{Conflicts of interest}

There are no conflicts of interest to declare.

\section{Acknowledgements}

We are thankful for the support of the Nature and Science Foundation Committee of China (No. 81473548). 


\section{References}

1 W. M. Loke, M. Proudfoot, J. M. Hodgson, A. J. Mckinley, N. Hime, M. Magat, R. Stocker and K. D. Croft, Arterioscler., Thromb., Vasc. Biol., 2010, 30, 749-757.

2 F. Helderman, D. Segers, R. Crom, B. P. Hierck, R. E. Poelmann, P. C. Evans and R. Krams, Curr. Opin. Lipidol., 2007, 18, 527-533.

3 V. Singh, R. L. Tiwari, M. Dikshit and M. K. Barthwal, Curr. Vasc. Pharmacol., 2009, 7, 75-109.

4 K. S. Heo, K. Fujiwara and J. Abe, Mol. Cells, 2014, 37, 435440.

5 P. Nigro, A. Jun-ichi and B. C. Berk, Antioxid. Redox Signaling, 2011, 15, 1405-1414.

6 X. Deng, Y. Marois, T. How, Y. Merhi, M. King, R. Guidoin and T. Karino, J. Vasc. Surg., 1995, 21, 135-145.

7 S. A. Berceli, V. S. Warty, R. A. Sheppeck, W. A. Mandarino, S. K. Tanksale and H. S. Borovetz, Arteriosclerosis, 1990, 10, 686-694.

8 A. Tuttolomondo, D. D. Raimondo, R. Pecoraro, V. Arnao and A. Pinto, Curr. Pharm. Des., 2012, 18, 4266-4288.

9 C. Cheng, D. Tempel, R. V. Haperen, A. V. Baan, F. Grosveld, M. J. Daemen, R. Krams and R. de Crom, Circulation, 2006, 113, 2744-2753.

10 M. Toda, K. Yamamoto, N. Shimizu, S. Obi and S. Kumagaya, J. Biotechnol., 2008, 133, 239-244.

11 A. M. Malek, R. Jackman, R. D. Rosenberg and S. Izumo, Circ. Res., 1994, 74, 852-860.

12 Y. Kawai, Y. Matsumoto, Y. Ikeda and K. Watanabe, Rinsho Byori, 1997, 45, 315-320.

13 H. L. Yuan, N. Deng, S. M. Zhang, Y. G. Cao, Q. Wang, X. Liu and Q. Zhang, J. Hematol. Oncol., 2012, 5, 65.

14 Y. Y. Hao, J. Cao, X. J. Yin, L. L. Gong, H. J. Yuan, L. Wang, R. X. Liang and F. L. Liao, Chinese Journal of Microcirculation, 2016, 26, 1-6.

15 W. Li, J. H. Jeong, H. G. Park, Y. R. Lee, M. Li and S. Lee, J. Exerc. Nutrition Biochem., 2014, 18, 105-110.

16 Y. You, W. H. Liu, Y. Li, Y. Zhang, D. F. Li, W. Li, X. J. Yin, F. L. Liao, X. X. Zhu and C. F. Liu, Clin. Hemorheol. Microcirc., 2011, 47, 187-198.

17 J. L. Wang, L. Wang, H. J. Yang, Y. You, H. Y. Xu, L. L. Gong, X. J. Yin, W. D. Wang, S. R. Gao, L. Cheng, R. X. Liang and F. L. Liao, BMC Complementary Altern. Med., 2015, 15, 109122.

18 J. Zhang, H. Yu, Y. Sheng, L. Li, M. Ye and D. Guo, Biomed. Chromatogr., 2005, 19, 15-18.

19 M. Song, T. J. Hang and Z. X. Zhang, Acta Pharm. Sin. B, 2007, 42, 301 .
20 Z. Zuo, L. Zhang, L. Zhou, Q. Chang and M. Chow, Life Sci., 2006, 79, 2455-2462.

21 J. Y. Zhang, R. Y. Yan, L. Wang, J. L. Wang, W. D. Wang, R. Hou, H. L. Liu, H. J. Yang, R. X. Liang and B. Yang, China J. Chin. Mater. Med., 2013, 38, 1988-1991.

22 S. F. Ding, M. Ni, X. L. Liu, L. H. Qi, M. Zhang, C. X. Liu and Y. A. Zhang, Am. J. Physiol.: Heart Circ. Physiol., 2010, 298, H2121-H2129.

23 D. Nam, C. W. Ni, A. Rezvan, S. Jin, K. Budzyn, A. Llanos, D. Harrison, D. Giddens and H. Jo, Am. J. Physiol.: Heart Circ. Physiol., 2009, 297, H1535.

24 F. Hua, J. Ma, Y. Li, T. Ha, Y. Xia, J. Kelley, D. L. Williams, I. W. Browder, J. B. Schweitzer and C. Li, Neurosci. Lett., 2006, 400(1-2), 69-74.

25 K. J. Livak and T. D. Schmittgen, Methods, 2001, 25, 402-408. 26 V. Singh, R. L. Tiwari and M. Dikshit, Curr. Vasc. Pharmacol., 2009, 7, 75.

27 A. Rezvan, C. W. Ni, N. Alberts-Grill and H. Jo, Antioxid. Redox Signaling, 2011, 15, 1433-1448.

28 W. M. Loke, J. M. Proudfoot, J. M. Hodgson, A. J. Mckinley, N. Hime, M. Magat, R. Stocker and K. D. Croft, Arterioscler., Thromb., Vasc. Biol., 2010, 30, 749-757.

29 R. Ross, N. Engl. J. Med., 1999, 340, 115-126.

30 H. Schuett, R. Oestreich, G. H. Waetzig, W. Annema, M. Luchtefeld, A. Hillmer, U. Bavendie, J. Felden, D. Divchev, T. Kempf, K. C. Wollert, D. Seegert, S. RoseJoh, U. Tietge, B. Schieffe and K. Grote, Arterioscler., Thromb., Vasc. Biol., 2012, 32, 281.

31 A. Cignarella, Atherosclerosis, 2011, 217, 64-66.

32 M. Mudau, A. Genis, A. Lochner and H. Strijdom, Cardiovasc. J. Afr., 2012, 23, 222.

33 J. Vane and R. E. Corin, Eur. J. Vasc. Endovasc. Surg., 2003, 26, 571-578.

34 H. S. Bassiouny, R. H. Song, H. Kocharyan, E. Kins and S. Glagov, J. Vasc. Surg., 1998, 27, 910.

35 H. Shankaran, P. Alexandridis and S. Neelamegham, Blood, 2003, 101, 2637-2645.

36 J. J. Chiu and C. Shu, Physiol. Rev., 2011, 91, 327-387.

37 F. J. Gijsen, J. J. Wentzel, A. Thury, B. Lamers, J. C. Schuurbiers, P. W. Serruys and A. F. van der Steen, J. Biomech., 2007, 40, 2349-2357.

38 S. M. Mccormick, S. G. Eskin, L. V. Mcintire, C. L. Teng, C. M. Lu, C. G. Russell and K. K. Chittur, Proc. Natl. Acad. Sci. U. S. A., 2001, 98, 8955-8960.

39 G. M. James and W. C. Hodgson, Immunol., Endocr. Metab. Agents Med. Chem., 2012, 12, 279-288.

40 F. L. Liao, M. Li, D. Han, J. Cao and K. J. Chen, Trends Pharmacol. Sci., 2006, 27, 287. 